\title{
A Fuzzy ANP Model Integrated with Benefits, Opportunities, Costs, and Risks to Prioritize Intelligent Power Grid Systems
}

\author{
Hsing Hung Chen and Hao Gu \\ Faculty of Management and Administration, Macau University of Science and Technology, Wei Long Avenue, Taipa, Macau \\ Correspondence should be addressed to Hao Gu; gu-hao@qq.com
}

Received 16 March 2013; Accepted 14 May 2013

Academic Editor: Tofigh Allahviranloo

Copyright ( $) 2013$ H. H. Chen and H. Gu. This is an open access article distributed under the Creative Commons Attribution License, which permits unrestricted use, distribution, and reproduction in any medium, provided the original work is properly cited.

\begin{abstract}
Although growth of renewable energy is envisaged, many concerns are critical like the ability to maintain the balance between demands and supply and the variability, noncontrollability, and flexibility of the sources. Then, what will be the future concerns about the main composition of intelligent power grid systems in the future? There is no such research tackled before. Thus, this paper first finds critical success criteria of intelligent power grid systems and then constructs a multiple criteria and decision making model to help in identifying the suitable trends under complex economic performance, environmental impacts, and rapid technological and marketing changes. After empirical demonstration, the paper summarizes that the most suitable composition of future intelligent power grid systems should be constituted by "DHT" P2P grid, "C\&D workflow" P2P scheduling, "GARCM" trustworthy P2P grid, and "multipurpose" grid applications in the future.
\end{abstract}

\section{Introduction}

One of the major problems about energy sources in China tends to result from geographical mismatch between demand and supply [1]. China plans to set up a unified power grid network until 2020 because it lacks a unified one across the nation. In addition, development and construction of power grid systems in China tend to be sluggish $[2,3]$. For the purpose of building up an extra-high-voltage power grid with the characteristics of long-distance and high-capacity transmission and approaching a robust grid system, the power grid system in the future should be based on current China's economic development and characteristics of geographical mismatch between supply and demand [4]. Further, Wu [5] stated that the main composition of intelligent power grid systems in the future will be upgraded from current powerfocused grids into synthesized and interactive grids with functionality of power management, intelligent home appliance, automation building, electric transportation, telecommunication management, and video entertainments [6]. In summary, the increased value from versatile services, the balance of demand and supply in the grid systems, and demand taking a more active role in matching electricity generation, are likely to be very significant in the future. Then, what will be the main concerns about intelligent power grid systems in the future? None of such empirical and theoretical research has ever been tackled before.

In order to challenge the unfilled problem, the paper first discusses critical success factors of intelligent power grid systems and then constructs multiple criteria and decision making (MCDM) models to help in identifying the most important components based on economic performance, social and marketing impacts, and rapid technological and marketing changes. Most MCDM models, such as conventional analytic network process (ANP), usually adopt pairwise comparison with respect to its network's criteria to rank its final priority. A fuzzy ANP (FANP) model is adopted to compensate the uncertain and obscure environment and human judgment. However, synthesizing the introducing positive criteria like benefits (B) and opportunities (O) and the introducing negative criteria like costs $(\mathrm{C})$ and risks $(\mathrm{R})$ with rating calculation like additive, probabilistic additive, subtractive, probabilistic subtractive, and multiplicative is a more instinctive method to a daily life. Accordingly, FANP with BOCR is applied in the paper to handle this kind of positive and negative criteria in public-oriented projects. 
After empirical demonstration, the paper finds that the most important composition of intelligent grid system in the future should be constituted by "DHT" P2P grid, "C\&D workflow" P2P scheduling, "GARCM" trustworthy P2P grid, and "multipurpose" grid applications. The contributions of the paper may include the following. (1) A conceptual decision-making model for prioritizing public-oriented projects is proposed. (2) The result will help bureaucrats, entrepreneurs, scientists, and other energy experts for identifying international standardization, projects, and potential partners in the area of grid technologies. (3) The result will also help policy makers quickly review surroundings and identify its potential to support energy strategy in the electricity infrastructure.

The rest of this paper is organized as follows. In Section 2, the previous literatures related to China's status and outlook of electricity are introduced. The intelligent power grid system and its critical success factors are examined in Section 3. An FANP model with BOCR for prioritizing projects related to intelligent power grid systems is constructed, and a real case is examined in Section 4. Some conclusions and discussions are provided in the last section.

\section{China's Status and Outlook of Electricity}

In recent years, the electric power industry of China has experienced a high-speed growth. During 2003 through 2008, the total installed capacity has doubled from $380 \mathrm{GW}$ to $793 \mathrm{GW}$ [7]. China's installed capacity of electric power was projected to exceed $900 \mathrm{GW}$ by 2010 and is likely to become the largest electric power system in the world in the future five years [8]. $705 \mathrm{GW}$ installed capacity in 2007 was made up of by $86 \%$ of thermal-based capacity, $12 \%$ of hydraulic, $2 \%$ of nuclear, and $2 \%$ of renewable energy [9]. The majority of the thermal capacity was comprised of coal-fired boilers with the efficiency far below international standard [10]. Carbon dioxide $\left(\mathrm{CO}_{2}\right)$ emissions of China were 5.85, 6.51, 7.01, 7.79, 8.27, 8.9, and 9.7 billion of tones from 2005 to 2011, respectively. According to the statistical data from The Netherlands Environmental Assessment Agency, $\mathrm{CO}_{2}$ emissions of China had surpassed those of the US in 2006, making China the largest carbon emitter [11].

China's proven reserves of anthracite and bituminous coals are 62,200 million metric tonnes (mmt) and those of subbituminous and lignite coals are $52,300 \mathrm{mmt}$ [12]. The total proven coal reserves account for $13.5 \%$ of total world stocks $[13,14]$. As of the end of 2007 , the ratio of reserves to production is 118 years. It is also widely known that China's distribution of coal is extremely uneven across regions.

China has many substantial rivers, more than 50,000 of which cover a basin area of over $100 \mathrm{~km}^{2}$, and 3886 of which have a hydropower potential of over $10 \mathrm{MW}$. However, the distribution of hydro resources is uneven [1]. There is a huge wind power potential in China, of around 700-1200 GW [15]. Wind power is expected to be the country's third largest power resource after coal and hydroelectric power by 2020 [16]. However, the geological distribution of wind power installed capacity is rather uneven.

More than two thirds of the country receive an annual solar radiation of more than $5000 \mathrm{MJ} / \mathrm{m}^{2}$ and more than
$2000 \mathrm{~h}$ of sunshine [17]. The total yield in China's solar cell in 2007 was $1088 \mathrm{MW}$, ranking it first in the world. Photovoltaic generation is projected to be $300 \mathrm{MW}$ by 2010 and $1.8 \mathrm{GW}$ by 2020, respectively, according to the Medium- and Long-Term Development Plan for Renewable Energy [18]. However, it is difficult to enable solar power generation to advance on a large scale until the technological advancement substantially reduces the cost of photovoltaic power generation. The installed nuclear power was 16.7, 50.5, and 86.3 terawatthours in 2000, 2005, and 2011, respectively [19]. Rapid growth in electricity demand and power structure adjustment has prompted acceleration of China's nuclear power plants construction. The government planned to increase nuclear generating capacity to $40 \mathrm{GW}$ by 2020 , with a further $18 \mathrm{GW}$ nuclear being under construction [1]. However, Chinese nuclear power development will face the bottleneck because of the supply of natural uranium fuel.

Though emissions of $\mathrm{CO}_{2}$ in China have been consecutively increasing, the ratio between emissions of $\mathrm{CO}_{2}$ and gross domestic production (GDP) has been decreased year by year. In addition, the installed renewable energy was 0.7 , 1.4, and 17.7 million tones oil equivalent in 2000, 2006, and 2011, respectively. However, energy problems in China are still critical because of the following reasons. China's energy sources tend to suffer from geographical mismatch between supply and demand, coal being concentrated in the North and Northwestern regions, hydro concentrated in the Southwest, and only nuclear energy being concentrated in the high usage area [13]. Construction and development of power grids in China tend to be sluggish. The seven individual grid systems run well but their interconnections are not, resulting in an insufficient intergrid electricity exchange capacity. The investment in generation capacity increased from US\$9.0 billion to US\$ 42.2 billion from 2002 to 2007 , but the investment in grid systems increased only from US\$19.0 billion to US\$ 32.3 billion at the same time [2]. China lacks a unified power grid network across the nation and plans to set one up by 2020 [3].

\section{The Intelligent Power Grid System and Its Critical Success Factors}

The objective of the future grid computing systems is the integration of heterogeneous computing and data resources with the target of providing a global computing space. A typical grid system is constituted by $\mathrm{P} 2 \mathrm{P}$ and grid computing.

Considering the tremendous and exponentially growing data generated by grid, with expected data rates of several terabytes a day and petabytes a year, data management methods by centralized and hierarchical grid reach their limits and may result in bottlenecks. Then, e-science communities investigate different technologies to provide fast access to the growing data sets. Considering their potential to provide high quality of service with low costs, Peerto-Peer (P2P) and Data Grid are two models that fit well these requirements. Regarding P2P, the structure of "Sector" provides support for persistent data storage, data sharing, and distributed data analysis for communities connected by wide area high speed networks [20]. Regarding Data Grid, 
scalable distributed hash tables (DHTs) and ontology-based information (DIS) organize resources into DHT ring based on VO mode [21]. The DIS exploits P2P DHT technique to improve the efficiency and scalability of grid information service.

Regarding grid scheduling, resources enter and leave at any time and the performance varies significantly over time, of which users have little or no knowledge. Then, a great challenge in grids is to build a scalable and efficient information service framework to support the initial discovery and ongoing monitoring of the existence and characteristics of resources contributed by different virtual organizations. The obtained results demonstrate that the prediction of process behavior is essential for an efficient scheduling in largescale and heterogeneous distributed environments. Then, by considering chaotic properties of such behavior and the automatic detection of critical execution points, the "e-science workflow" is applied and evaluated for process scheduling in cluster and grid computing environments by Rahman et al. [22]. Oppositely, another approach derives from a DHT-based $d$-dimensional logical index space with regards to resource discovery, coordination, and overall system decentralization and is called Cooperative and Decentralized Workflow Scheduling in Global Grids (CDworkflow) [23].

Virtualization technologies have become popular, which allows one computer system to function as multiple virtual systems. When a P2P grid node is equipped with virtualization technologies, the virtual machine monitor (VMM) is more secure than the operating systems (OS) because the VMM is much simplified than the OS, and trusted platform module (TPM) embedded into the underlying hardware can provide integrity protection for the VMM. On the other hand, because of heterogeneous properties, volunteers (resource providers) in $\mathrm{P} 2 \mathrm{P}$ grid used to dynamically join and leave during execution. Some volunteers may behave erratically or maliciously. Thus, it is important to detect and tolerate the erroneous results in order to guarantee a reliable execution. Then, a trusted execution environment on P2P grid nodes equipped with secure VMM is proposed [24]. A VMM image used for deploying virtual execution environment will be selected and deployed onto a P2P grid node according to the job requirement and node situation, such as node performance and node reputation. Oppositely, a new GroupBased Adaptive Result Certification Mechanism (GARCM) is proposed to dynamically perform result certification and scheduling algorithms to each group according to its properties such as volunteering service time, availability, and credibility [25]. Consequently, the GARCM can reduce the overhead and latency and therefore complete more tasks while guaranteeing reliable results.

The grid applications layer is typically developed using the components of user level middleware. This layer supports users to execute their applications on remote resources and collect results from them using web portals or applications such as the Grid Application Toolkit (GAT) and java Commodity Grid kit (CoG) [26, 27]. There are two grid applications including single-purpose and multi-purpose grids. Many of the current grid users are "grid hackers" or single-purpose grid. If the grid wants to be a part of real life, it should involve further users and user communities who do not have the previously mentioned knowledge. They want to migrate and deploy their calculation, modeling, simulation, and applications on the grid, to run them with minimum costs and efforts having access to grid resources in a transparent way. It is called a multi-purpose grid.

Based on extensive literatures [20-29] and the evaluation of the committee, the most important factors for successful grid systems are as follows. Under benefits (B), there are criteria: functionality (b1) including availability, efficiency, and openness and standardization; reliability (b2) including accuracy, quality, and stability; and usability (b3) like ease of use, easy to repair, and easy fault identification. The criteria under opportunities (O) are scaling capabilities (o1) including easy expansion and extension in hardware and software and in numbers of sites and suppliers; learning \& innovation (o2) including easy access, learning, and sharing of technology and information; and flexibility (o3) including compatibility of hardware support, good application software to provide ability to interface, support and manage files and to perform storage, retrieval, manipulation, and transmission functions. Under costs (C) there are criteria: product price (c1) including purchase, installation, and maintenance for hardware and software; R\&D spending (c2) like hardware and software expenditure; and foundation spending (c3) including the spending incurring from main building construction, backbone construction, and grid connection. The criteria under risks $(\mathrm{R})$ are concept conflict ( $\mathrm{rl}$ ) like conflicts among entrepreneurs, policy makers, and other stakeholders; technical risks (r2) like inadequacy in advanced technologies; and cultural differences and geographical mismatch (r3). In order to select the best composition of future grid projects to develop in the subsequent sections, the authors constructed an FANP model with BOCR for a real case study.

\section{An FANP Model with BOCR for a Real Case Study}

The purpose of the section is to employ the proposed systematic FANP model with BOCR to identify future intelligent energy grid systems by comprehensively qualitative and quantitative analyses of the economic, environmental, and technical performance of alternatives.

At the beginning stage, eleven experts, including bureaucrats, energy system planners, energy grid researchers, and other stakeholders, contributed their professional experience and formed the evaluation committee. Totally, there are sixteen alternatives; however, only six combinations alternatives, shown in Table 1, passed the first-round selection by the evaluation committee, and they are represented as combinations A, B, C, D, E, and F. A questionnaire is constructed, and the members of the evaluation committee are invited to contribute their professional experience. Based on the collected opinions of the experts and the proposed model, the performance of the six combinations can be generated.

Multicriteria analysis is a set of methods that are designed to handle problems characterized by multiple objectives. The methodologies typically aim at quantifying tradeoffs 
TABLE 1: Different combinations for six alternatives.

\begin{tabular}{lcccc}
\hline Systems alternatives & P2P grid & P2P scheduling & Trustworthy P2P grid & Grid application \\
\hline Combination A & DHT & C\&D workflow & VMM & Multipurpose \\
Combination B & Sector & C\&D workflow & GARCM & Single purpose \\
Combination C & DHT & e-Science workflow & GARCM & Multi-purpose \\
Combination D & DHT & C\&D workflow & VMM & Multi-purpose \\
Combination E & Sector & e-Science workflow & Single purpose \\
Combination F & DHT & e-Science workflow & Single purpose \\
\hline
\end{tabular}

TABLE 2: Characteristic function of the fuzzy numbers.

\begin{tabular}{lc}
\hline Fuzzy number & Characteristic (membership) function \\
\hline$\widetilde{1}$ & $(1,1,3)$ \\
$\widetilde{x}$ & $(x-2, x, x+2)$ for $x=3,5,7$ \\
$\widetilde{9}$ & $(7,9,9)$ \\
$1 / \widetilde{1}$ & $\left(3^{-1}, 1^{-1}, 1^{-1}\right)$ \\
$1 / \widetilde{x}$ & $\left((x+2)^{-1}, x^{-1},(x-2)^{-1}\right)$ for $x=3,5,7$ \\
$1 / \widetilde{9}$ & $\left(9^{-1}, 9^{-1}, 7^{-1}\right)$ \\
\hline
\end{tabular}

among attributes. How this is done and the requirements for elicitation of weighting factors vary between methods [30]. Based on Saaty and Lee [31-34], an FANP model with BOCR $[35,36]$ for prioritizing intelligent power grid systems is proposed. The procedures are summarized as follows.

Step 1. Decompose the problem into a control hierarchy with control criteria, as in Figure 1, for determining the priorities of the merits, benefits (B), opportunities (O), costs $(\mathrm{C})$, and risks $(\mathrm{R})$. The goal of the control hierarchy is to calculate the relative importance of the four merits [37].

The relationship can be divided into two networks: the control network and the BOCR network. The first level of the control network contains the goal, the selection of the best suitable project. In the second level, four strategic criteria are considered: social and economic performance (CC1), financial and marketing performance (CC2), legal and environmental performance (CC3), and technical and manufacturing performance (CC4). In the third level, there are benefits (B), opportunities (O), costs (C), and risks (R) four merits. The purpose of the control network is to calculate the priorities of the four merits.

Step 2. Decompose the problem into a BOCR network. Based on the literature review and experts' opinions, a network in Figure 2 is constructed. Four merits, which reflect both positive and negative impacts of selecting a particular alternative, must be considered in achieving the overall goal. A subnetwork is formed for each of the merits. For instance, for the sub-network for benefits merit, there are criteria for achieving the benefits, and the lowest level contains the alternatives.

Their first task was to select critical success criteria, as described in Section 3. The relationship of future grid project among final goal, strategic criteria, merits, sub-criteria, and alternatives is structured by evaluation committee as shown in Figure 2. The BOCR network can be further divided into four sub-networks including benefits, opportunities, costs, and risks. Under merit benefits, there are three criteria, group factors (b1) through (b3). Under merit opportunities, there are three detailed criteria, group factors (o1), (o1), and (o3). Group factors (c1), (c2), and (c3) are the criteria of merit costs, and group factors (r1), (r2), and (r3) are the criteria of merit risks.

Step 3. Formulate a questionnaire based on the proposed control hierarchy and BOCR network to pairwise compare elements.

Experts in the field are asked to fill out the ninepoint-scale questionnaire. For benefits and opportunities, the question is to ask what gives the most benefit or presents the greatest opportunity to influence fulfillment of the criterion. For costs and risks, the question is to ask what incurs the most cost or faces the greatest risk. The consistency property of each expert's comparison results is examined first. If an inconsistency is found in an expert's result, the expert is asked to revise the inconsistent part of the questionnaire until a consistency is met.

Step 4. Calculate crisp relative importance weights for control criteria for attaining the goal. From each expert's questionnaire result, establish fuzzy importance weights for control criteria according to the membership functions in Table 2. A triangular fuzzy number $\widetilde{D}$ is obtained by combining the experts' opinions using the geometric mean method. Consider

$$
\widetilde{D}=\left(n^{-}, n, n^{+}\right)
$$

where

$$
\begin{aligned}
& n^{-}=\left(\prod_{t=1}^{s} l_{t}\right)^{1 / s}, \quad \forall t=1,2, \ldots, s, \\
& n=\left(\prod_{t=1}^{s} m_{t}\right)^{1 / s}, \quad \forall t=1,2, \ldots, s, \\
& n^{+}=\left(\prod_{t=1}^{s} u_{t}\right)^{1 / s}, \quad \forall t=1,2, \ldots, s,
\end{aligned}
$$




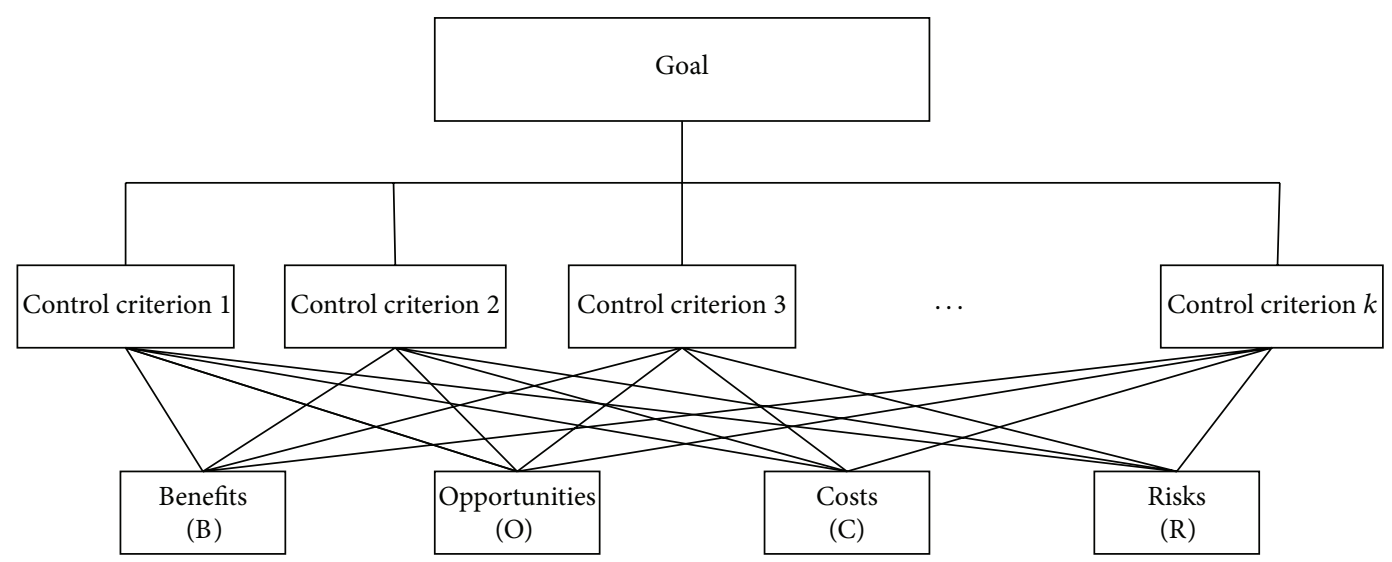

FIgURE 1: The control hierarchy.

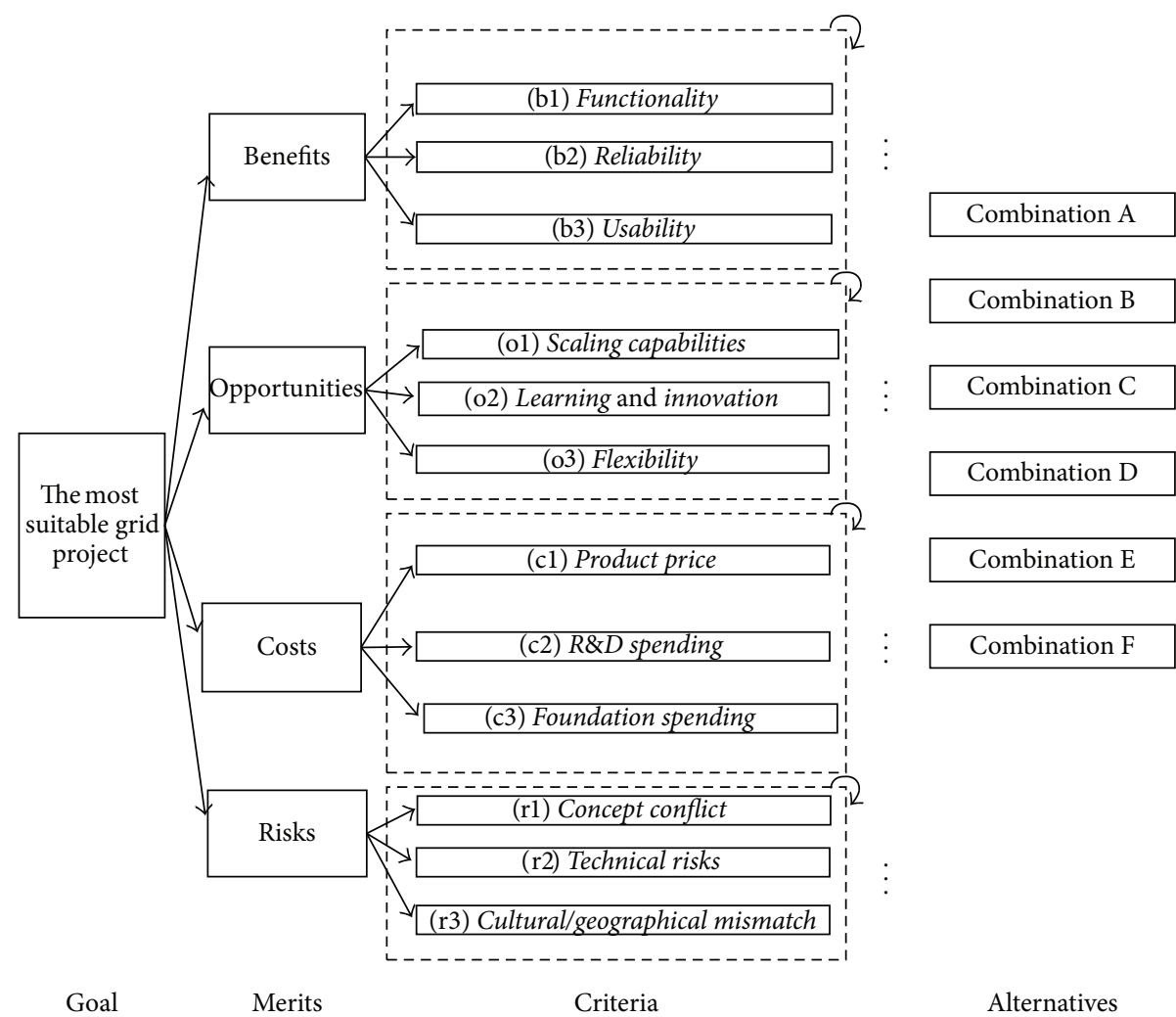

FIGURE 2: The BOCR network for selecting intelligent grid power systems.

and $\left(l_{t}, m_{t}, u_{t}\right)$ is the importance weight from expert $t$. Apply the center of gravity (COG) method to defuzzify the triangular fuzzy numbers [37]

$$
n^{*}=\frac{\left[\left(n^{+}-n^{-}\right)+\left(n-n^{-}\right)\right]}{3}+n^{-}=\frac{n^{-}+n+n^{+}}{3} .
$$

Use the defuzzificated values to form pairwise comparison matrix for control criteria. Calculate the maximum eigenvalue, $\lambda_{\text {max }}$, and the eigenvector for the matrix [31]

$$
\mathbf{A} \cdot w=\lambda_{\max } \cdot w \text {. }
$$

In the first part of the model, experts are asked to evaluate the priorities of benefits, opportunities, costs, and risks. After pairwise comparison matrices of control criteria from all experts passed the consistency test, the fuzzy pairwise comparison matrices are aggregated into one single fuzzy pairwise comparison matrix through the geometric mean method. For example, the fuzzy pairwise comparison values between CC1 and CC2 for the experts were $\widetilde{3}^{-1}, \widetilde{5}^{-1}, \widetilde{3}, \widetilde{1}, \widetilde{3}^{-1}, \widetilde{1}$, $\widetilde{5}^{-1}, \widetilde{3}^{-1}, \widetilde{3}$ and $\widetilde{3}^{-1}$, that is, $(1 / 5,1 / 3,1),(1 / 7,1 / 5,1 / 3),(1,3,5)$, $(1,1,3),(1 / 5,1 / 3,1),(1,1,3),(1 / 7,1 / 5,1 / 3),(1 / 5,1 / 3,1),(1,3,5)$, and $(1 / 5,1 / 3,1)$. The fuzzy aggregated paiwise comparison value between $\mathrm{CC} 1$ and CC2 was $(0.356,0.582,1.380)$, and 
TABLE 3: Comparison matrix for the control criteria.

\begin{tabular}{lccccc}
\hline & CC1 & CC2 & CC3 & CC4 & Priorities of control criteria \\
\hline CC1 & 1 & 0.772 & 3.195 & 5.525 & 0.371 \\
CC2 & 1.295 & 1 & 4.717 & 4.219 & 0.425 \\
CC3 & 0.313 & 0.212 & 1 & 4.065 & 0.140 \\
CC4 & 0.181 & 0.237 & 0.246 & 1 & 0.063 \\
\hline
\end{tabular}

$\lambda_{\max }=4.202 ; \mathrm{CI}=0.068 ; \mathrm{CR}=0.073$.

TABLE 4: Weights of the four merits.

\begin{tabular}{|c|c|c|c|c|c|c|c|c|c|}
\hline & \multicolumn{2}{|c|}{ CC1 $(0.371)$} & \multicolumn{2}{|c|}{ CC2 (0.425) } & \multicolumn{2}{|c|}{ CC3 $(0.140)$} & \multicolumn{2}{|c|}{ CC4 (0.063) } & \multirow{2}{*}{$\begin{array}{l}\text { Relative } \\
\text { importance } \\
\text { weights }\end{array}$} \\
\hline & $\begin{array}{c}\text { Crisp } \\
\text { weights }\end{array}$ & $\begin{array}{c}\text { Normalized } \\
\text { weights }\end{array}$ & $\begin{array}{c}\text { Crisp } \\
\text { weights }\end{array}$ & $\begin{array}{l}\text { Normalized } \\
\text { weights }\end{array}$ & $\begin{array}{c}\text { Crisp } \\
\text { weights }\end{array}$ & $\begin{array}{l}\text { Normalized } \\
\text { weights }\end{array}$ & $\begin{array}{c}\text { Crisp } \\
\text { weights }\end{array}$ & $\begin{array}{l}\text { Normalized } \\
\text { weights }\end{array}$ & \\
\hline Benefits (B) & 6.852 & 0.268 & 7.482 & 0.314 & 6.754 & 0.289 & 6.754 & 0.259 & 0.289 \\
\hline Opportunities (O) & 7.743 & 0.303 & 7.235 & 0.303 & 7.237 & 0.310 & 7.758 & 0.297 & 0.303 \\
\hline Costs $(\mathrm{C})$ & 8.035 & 0.314 & 6.529 & 0.274 & 5.723 & 0.245 & 5.873 & 0.225 & 0.282 \\
\hline Risks (R) & 2.927 & 0.115 & 2.574 & 0.109 & 3.635 & 0.156 & 5.726 & 0.219 & 0.126 \\
\hline
\end{tabular}

the crisp value is 0.772 . The fuzzy aggregated pairwise comparison matrix of control criteria was formed, and it was then defuzzified into aggregated pairwise comparison matrices using the COG method. The priorities of control criteria were calculated, as shown in Table 3.

Step 5. Calculate crisp relative importance weights for merits with respect to each control criterion. A five-level scale is used, and the linguistic term and the triangular fuzzy number of each scale is assigned to be very high, $(7,9,9)$; high, $(5,7,9)$; medium, $(3,5,7)$; low, $(1,3,5)$; and very low, $(1,1,3)$. Aggregate experts' opinions by the geometric mean method, and apply Centroid method to defuzzify the fuzzy numbers.

The importance of each merit to each control criterion was determined next. For instance, the experts' ratings of benefits on CC1 were "high," "very high," "medium," "high," "high," "very high," "high," "high," "medium," and "high." The fuzzy numbers were $(5,7,9),(7,9,9),(3,5,7),(5,5,9),(5,7,9)$, $(7,7,9),(5,9,9),(5,7,9),(3,5,7)$, and $(5,7,9)$. The aggregated triangular fuzzy number was $(5.174,6.873,8.5442)$, and the crisp value was 6.852 . All the calculated weights, the normalized weights of the four merits on control criteria, and the relative importance of control criteria are shown in Table 4.

Step 6. Calculate relative importance weights, b, o, c, and r, for the four merits B, O, C, and R, by synthesizing the importance vector of control criteria from Step 4 and the importance vectors for merits with respect to each criterion from Step 5.

Step 7. Calculate crisp relative importance weights for criteria with respect to the same merit, the interdependence of the criteria with respect to the same merit, and relative priorities of the alternatives with respect to each criterion, using a similar procedure in Step 4.

Step 8. Calculate the priorities of the alternatives for each merit sub-network. Form an unweighted supermatrix for each sub-network using the local priority vectors obtained from Step 7. Calculate the weighted supermatrix and the limit supermatrix for each merit sub-network. The priorities of the alternatives under a merit are obtained in the alternative-togoal column of the limit supermatrix of the merit.

In the second part of the model, the priorities of the alternatives under each merit were calculated. The relative importance weights of criteria with respect to the same upper level merit and the interdependence priorities among the criteria that have the same upper-level merit were calculated. The performance results of different alternatives under various criteria, however, were collected from each expert individually in order to limit the number of pairwise comparisons. All criteria including quantitative and qualitative variables were rated in a range from zero to a hundred. The synthesized performance value of each alternative on each criterion was calculated by geometric averaging the results from all the experts. These performance values were further transformed into a number between zero and one by dividing the performance value of an alternative on a criterion by the largest performance value among all alternatives on the same criterion. An unweighted supermatrix was formed for each merit, and that for the benefits merit is shown in Table 5. The overall performance of each alternative under each merit was obtained after calculating the limit supermatrices, and the results are shown in Table 6.

Step 9. Calculate overall priorities of alternatives by synthesizing priorities of each alternative under each merit from Step 8 with corresponding merit priority weights, b, o, c and $r$, from Step 6. There are five ways: additive, probabilistic additive, subtractive, multiplicative, and multiplicative priority powers $[32,34]$.

The final rankings of the alternatives are calculated by the five methods to combine the scores of each alternative under B, O, C, and R. The results are as shown in Table 7. Under all five methods of synthesizing the scores of alternatives, combination $\mathrm{D}$ ranks the first. While combinations $\mathrm{B}, \mathrm{E}$ and $\mathrm{F}$ always stay, respectively, as the fourth, fifth, 
TABLE 5: Unweighted supermatrix for benefits subnetwork.

\begin{tabular}{lcccccccccc}
\hline & Benefits (B) & b1 & b2 & b3 & Comb. A & Comb. B & Comb. C & Comb. D & Comb. E & Comb. F \\
\hline Benefits (B) & 0.5 & 0 & 0 & 0 & 0 & 0 & 0 & 0 & 0 \\
b1 & 0.20265 & 0.14576 & 0.20934 & 0.23674 & 0 & 0 & 0 & 0 & 0 \\
b2 & 0.11371 & 0.11348 & 0.08721 & 0.05471 & 0 & 0 & 0 & 0 & 0 \\
b3 & 0.18364 & 0.07861 & 0.08647 & 0.11358 & 0 & 0 & 0 & 0 & 0 & 0 \\
\hline Combination A & 0 & 0.09083 & 0.10369 & 0.10351 & 1 & 0 & 0 & 0 & 0 \\
Combination B & 0 & 0.07657 & 0.07525 & 0.07433 & 0 & 1 & 0 & 0 & 0 \\
Combination C & 0 & 0.10932 & 0.09862 & 0.08243 & 0 & 0 & 1 & 0 & 0 \\
Combination D & 0 & 0.12762 & 0.11786 & 0.12891 & 0 & 0 & 0 & 1 & 0 \\
Combination E & 0 & 0.04761 & 0.05422 & 0.06541 & 0 & 0 & 0 & 0 & 0 \\
Combination F & 0 & 0.05743 & 0.07845 & 0.08502 & 0 & 0 & 0 & 0 & 0 \\
\hline
\end{tabular}

TABLE 6: Priorities of alternatives under four merits.

(a)

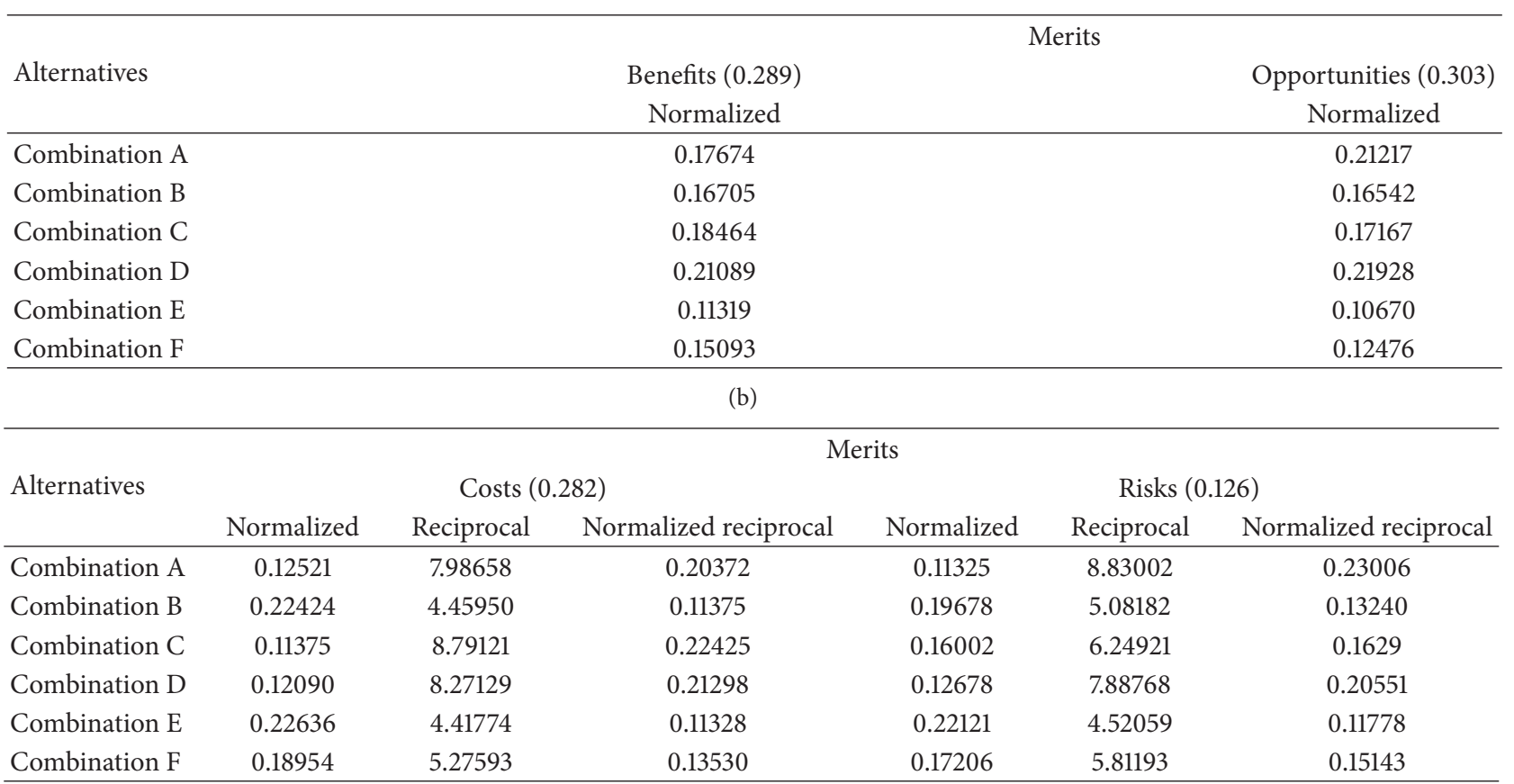

TABLE 7: Final synthesis of priorities of alternatives.

\begin{tabular}{|c|c|c|c|c|c|c|c|c|c|c|}
\hline \multirow{3}{*}{ Alternatives } & \multicolumn{10}{|c|}{ Synthesizing methods } \\
\hline & \multicolumn{2}{|c|}{ Additive } & \multicolumn{2}{|c|}{ Probabilistic additive } & \multicolumn{2}{|c|}{ Subtractive } & \multicolumn{2}{|c|}{ Multiplicative priority powers } & \multicolumn{2}{|c|}{ Multiplicative } \\
\hline & Priority & Rank & Priority & Rank & Priority & Rank & Priority & Rank & Priority & Rank \\
\hline Combination $\mathrm{A}$ & 0.2050 & 2 & 0.4238 & 3 & 0.0434 & 3 & 0.2053 & 2 & 1.1542 & 2 \\
\hline Combination B & 0.1703 & 4 & 0.4117 & 4 & 0.0303 & 4 & 0.1510 & 4 & 0.8663 & 4 \\
\hline Combination $\mathrm{C}$ & 0.2050 & 2 & 0.4472 & 2 & 0.0461 & 2 & 0.2035 & 3 & 1.0537 & 3 \\
\hline Combination $\mathrm{D}$ & 0.2121 & 1 & 0.4523 & 1 & 0.0526 & 1 & 0.2118 & 1 & 1.1783 & 1 \\
\hline Combination E & 0.0764 & 6 & 0.4013 & 6 & 0.0265 & 6 & 0.1011 & 6 & 0.7632 & 6 \\
\hline Combination $\mathrm{F}$ & 0.1312 & 5 & 0.4057 & 5 & 0.0282 & 5 & 0.1273 & 5 & 0.8035 & 5 \\
\hline
\end{tabular}


and the last contractors, combination $\mathrm{A}$ and combination $\mathrm{C}$ take turns in the ranking of the second and the third. Under multiplicative priority powers, as well as multiplicative methods, combination $\mathrm{A}$ is the second best, and combination $\mathrm{C}$ is the third. However, under probabilistic additive and subtractive methods, the opposite is true. In addition, under additive method, both combination $\mathrm{A}$ and combination $\mathrm{C}$ have the same ranking of the second best. Such a result is because the overall performances of the two combinations are very similar, and the final scores of the two combinations are not significantly different under all the methods of calculation. In addition, a comparison of the performances between combination $\mathrm{D}$ and $\mathrm{A}$ shows that combination $\mathrm{D}$ performs better in both the benefits and opportunities merits, but the opposite is true in the costs and risks merits. The final priorities of combination D and A under multiplicative method are 1.1783 and 1.1542 , respectively. Such performances are not significantly different. However, note that due to the nature of the multiplicative method, the importance of the four merits (B, O, C, and R) does not take effect in this calculation. Taking into account the different importance of the four merits in the other four calculation methods, combination D performs significantly better than combination $\mathrm{A}$ because combination $\mathrm{D}$ makes higher scores in the benefits and opportunities merits. Combination D, the most suitable composition of future intelligent grid system, is constituted by "DHT" P2P grid, "C\&D workflow" P2P scheduling, "GARCM" trustworthy P2P grid, and "multipurpose" grid applications.

\section{Discussion and Conclusion}

Renewable energy and other low-carbon energy sources will become major contributors to the electricity generation systems for the purpose of significant reduction in $\mathrm{CO}_{2}$ emissions. However, the flexibility, variability, noncontrollability, of the sources, and the ability to maintain the balance between demands and supply are difficult [38]. Under this kind of situation, the current power infrastructures in both Europe and America are getting out of date even with very different policies, as well as social and economical contexts. Key solutions for both of them seem to be the evolution toward a more intelligent power grid. The vision of the United States is mainly related to a largely centralized power grid system with a high integration of demand response and some penetration of distributed power systems used for additional support to the system [39]. Oppositely, the vision of Europe is clearly impacted by the concern derived from the wide range of natures and degrees of evolution of the power grids across European countries. The consequence of this concern is identified in the need to have a flexible, reliable and accessible power grid system. The future power grid systems in Europe can satisfy the needs of a wide range of customers and economies [39]. However, as the chief officer of the National Electric Company summarized, due to the differences in national condition, the main composition of future intelligent power grid systems between China and western nations should differ considerably in aspects including development procedure, development direction, and focal points. Then, the results of our estimated power grid systems are consistent with the well-known discussion.

No research article ever examines the main composition of future intelligent power grid systems by comprehensively qualitative and quantitative analyses of the alternatives. In addition, because of increasing complexity in the social and economic environments along with rapid technology changes and market demands, adopting MCDM to select the best project has a great potential since it does not only consider the price, but also concern various facets of projects. Especially, when a public project has positive criteria like benefits and opportunities and negative criteria like costs and risks, and an evaluation method has been exposed in an uncertain and vague environment in a daily life, FANP with BOCR is proposed to replace conventional ANP since it is a more instinctive evaluation model.

\section{References}

[1] Q. Wang and Y. Chen, "Status and outlook of China's freecarbon electricity," Renewable and Sustainable Energy Reviews, vol. 14, no. 3, pp. 1014-1025, 2010.

[2] J. Fenby and D. Qu, China's Grid Power Up, Trusted Sources UK Ltd, London, UK, 2008.

[3] People's Daily, China to create nationwide power Grid network by 2020, People's Daily, 2000.

[4] China Energy Network, "What is smart grid of China?" The data was downloaded from, 2010, http://baike.baidu.com/ view/2445516.htm.

[5] J. D. Wu, “The future smart grid in China," 2009, http://finance .sina.com.cn/chanjing/yjsy/20090805/23516576506.shtml.

[6] China Power Network, "What is smart Grid in China," The data was downloaded from the web, 2012, http://www.iotonline.com/xingyeyingyong/sg/.

[7] China Electricity Council, Statistical Bulletin of the National Electric Power Industry in 2007, China Electricity Pressing, Beijing, China, 2008.

[8] Z.-M. Jiang, "Reflections on energy issues in China," Journal of Shanghai Jiaotong University, vol. 13, no. 3, pp. 257-274, 2008.

[9] LBRL, China Energy Database, Lawrence Berkeley Research Laboratory, 2008.

[10] P. A. Steenhof, "Decomposition for emission baseline setting in China's electricity sector," Energy Policy, vol. 35, no. 1, pp. 280294, 2007.

[11] C. Ma and L. He, "From state monopoly to renewable portfolio: restructuring China's electric utility," Energy Policy, vol. 36, no. 5, pp. 1697-1711, 2008.

[12] British Petroleum, British Petroleum Statistical Review of World Energy, 2008.

[13] H. Ma, L. Oxley, and J. Gibson, "China's energy situation in the new millennium," Renewable and Sustainable Energy Reviews, vol. 13, no. 8, pp. 1781-1799, 2009.

[14] W. Yang, J. Kwon, N. Y. Chong, and Y. Oh, "Biologically inspired robotic arm control using an artificial neural oscillator," Mathematical Problems in Engineering, vol. 2010, Article ID 107538, 16 pages, 2010.

[15] J. Li, H. Gao, P. Shi, J. Shi, L. Ma, and H. Qing, China Wind Power Report in 2007, China Environmental Science Press, Beijing, China, 2007. 
[16] J. Li, Wind Force 12 in China, Chemical Industry Press,, Beijing, China, 2005.

[17] "CWERA, Center for wind and solar energy resources assessment,” 2009, http://cwera.cma.gov.cn/Website/index.php? ChannelID $=39 \&$ NewsID.

[18] NDRC, Medium- and Long-Term Nuclear Development Plan for Renewable Energy, National Development and Reform Commission, Beijing, China, 2007.

[19] World Nuclear Association and Nuclear Power in China, 2009, http://www.world-nuclear.org/info/inf63.html.

[20] Y. Tao, H. Jin, S. Wu, and X. Shi, "Scalable DHT- and ontologybased information service for large-scale grids," Future Generation Computer Systems, vol. 26, no. 5, pp. 729-739, 2010.

[21] E. Dodonov and R. F. de Mello, "A novel approach for distributed application scheduling based on prediction of communication events," Future Generation Computer Systems, vol. 26, no. 5, pp. 740-752, 2010.

[22] M. Rahman, R. Ranjan, and R. Buyya, "Cooperative and decentralized workflow scheduling in global grids," Future Generation Computer Systems, vol. 26, no. 5, pp. 753-768, 2010.

[23] D. Zou, W. Zheng, J. Long, H. Jin, and X. Chen, "Constructing trusted virtual execution environment in P2P grids," Future Generation Computer Systems, vol. 26, no. 5, pp. 769-775, 2010.

[24] S. Choi and R. Buyya, "Group-based adaptive result certification mechanism in Desktop Grids," Future Generation Computer Systems, vol. 26, no. 5, pp. 776-786, 2010.

[25] H. Izakian, A. Abraham, and B. T. Ladani, "An auction method for resource allocation in computational grids," Future Generation Computer Systems, vol. 26, no. 2, pp. 228-235, 2010.

[26] W. Yajuan and W. Xianjia, "A novel double auction mechanism based resource allocation in the Grid," in Proceedings of the International Conference on Management of e-Commerce and eGovernment (ICMeCG '09), pp. 420-423, September 2009.

[27] U. Schwiegelshohn, R. M. Badia, M. Bubak et al., "Perspectives on grid computing," Future Generation Computer Systems, vol. 26, no. 8, pp. 1104-1115, 2010.

[28] A. Haque, S. M. Alhashmi, and R. Parthiban, "A survey of economic models in grid computing," Future Generation Computer Systems, vol. 27, no. 8, pp. 1056-1069, 2011.

[29] S. Ahlroth, M. Nilsson, G. Finnveden, O. Hjelm, and E. Hochschorner, "Weighting and valuation in selected environmental systems analysis tools-suggestions for further developments," Journal of Cleaner Production, vol. 19, no. 2-3, pp. 145-156, 2011.

[30] T. L. Saaty, The Analytic Hierarchy Process, McGraw-Hill, New York, NY, USA, 1980.

[31] T. L. Saaty and M. Ozdemir, "Negative priorities in the analytic hierarchy process," Mathematical and Computer Modelling, vol. 37, no. 9-10, pp. 1063-1075, 2003.

[32] T. L. Saaty, "Fundamentals of the analytic network processmultiple networks with benefits, opportunities, costs and risks," Journal of Systems Science and Systems Engineering, vol. 13, no. 3, pp. 348-379, 2004.

[33] A. H. I. Lee, H. H. Chen, and H.-Y. Kang, "Multi-criteria decision making on strategic selection of wind farms," Renewable Energy, vol. 34, no. 1, pp. 120-126, 2009.

[34] Ş. Erdoğmuş, M. Kapanoglu, and E. Koç, "Evaluating high-tech alternatives by using analytic network process with BOCR and multiactors," Evaluation and Program Planning, vol. 28, no. 4, pp. 391-399, 2005.

[35] Ş. Erdoğmuş, H. Aras, and E. Koç, "Evaluation of alternative fuels for residential heating in Turkey using analytic network process (ANP) with group decision-making," Renewable and Sustainable Energy Reviews, vol. 10, no. 3, pp. 269-279, 2006.

[36] T. L. Saaty and J. S. Shang, "Group decision-making: headcount versus intensity of preference," Socio-Economic Planning Sciences, vol. 41, no. 1, pp. 22-37, 2007.

[37] R. R. Yagar, "On a general class of fuzzy connectives," Fuzzy Sets and Systems, vol. 4, pp. 235-242, 1980.

[38] G. Strbac, "Demand side management: benefits and challenges," Energy Policy, vol. 36, no. 12, pp. 4419-4426, 2008.

[39] D. Coll-Mayor, M. Paget, and E. Lightner, "Future intelligent power grids: analysis of the vision in the European Union and the United States," Energy Policy, vol. 35, no. 4, pp. 2453-2465, 2007. 


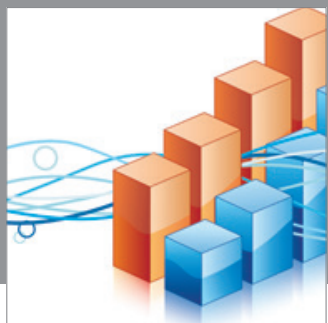

Advances in

Operations Research

mansans

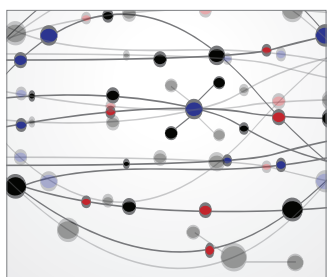

The Scientific World Journal
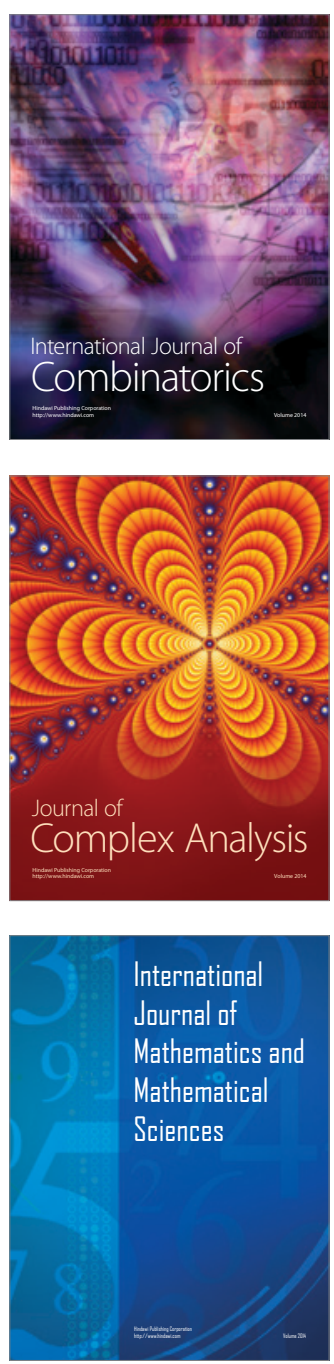
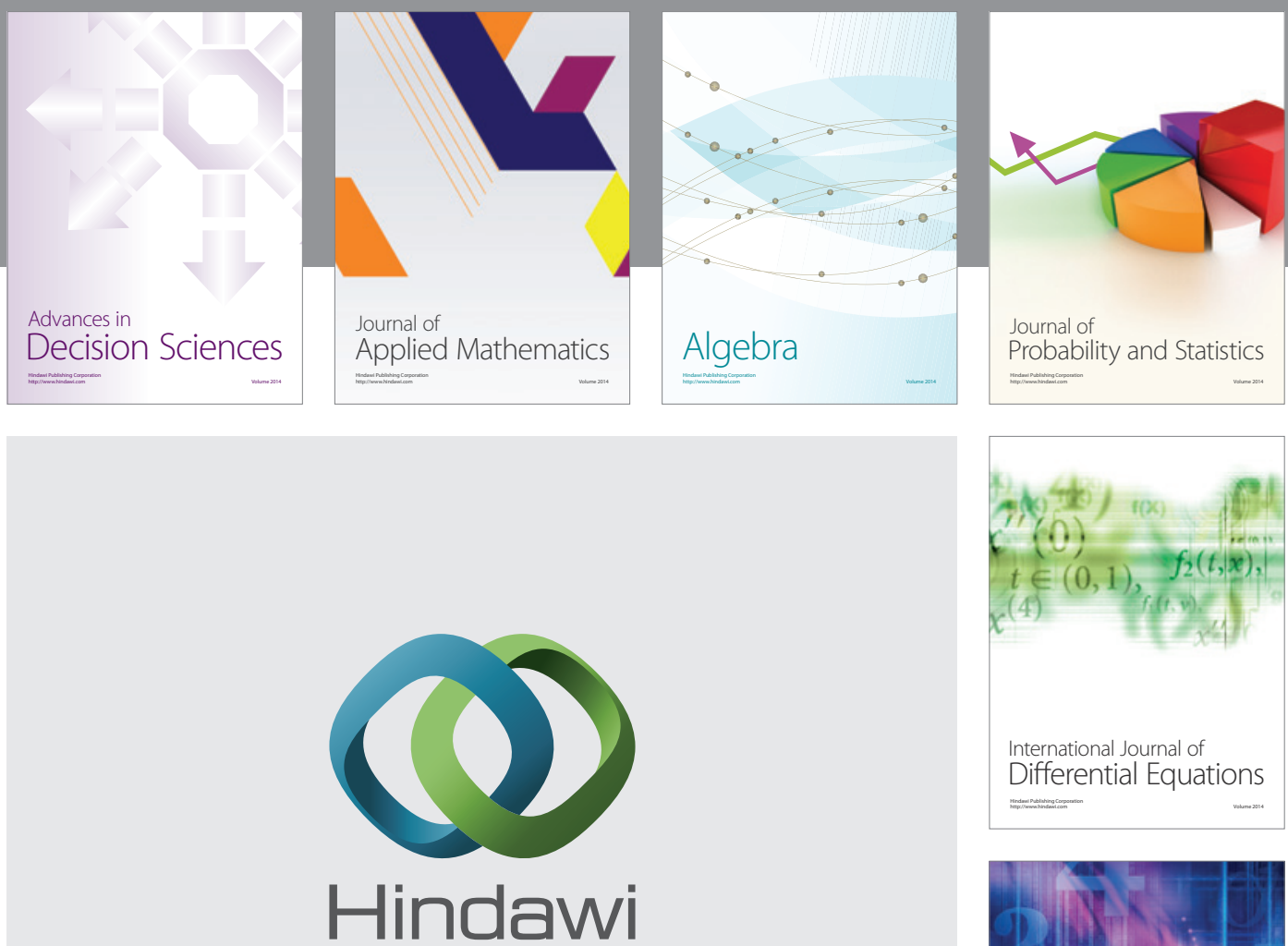

Submit your manuscripts at http://www.hindawi.com
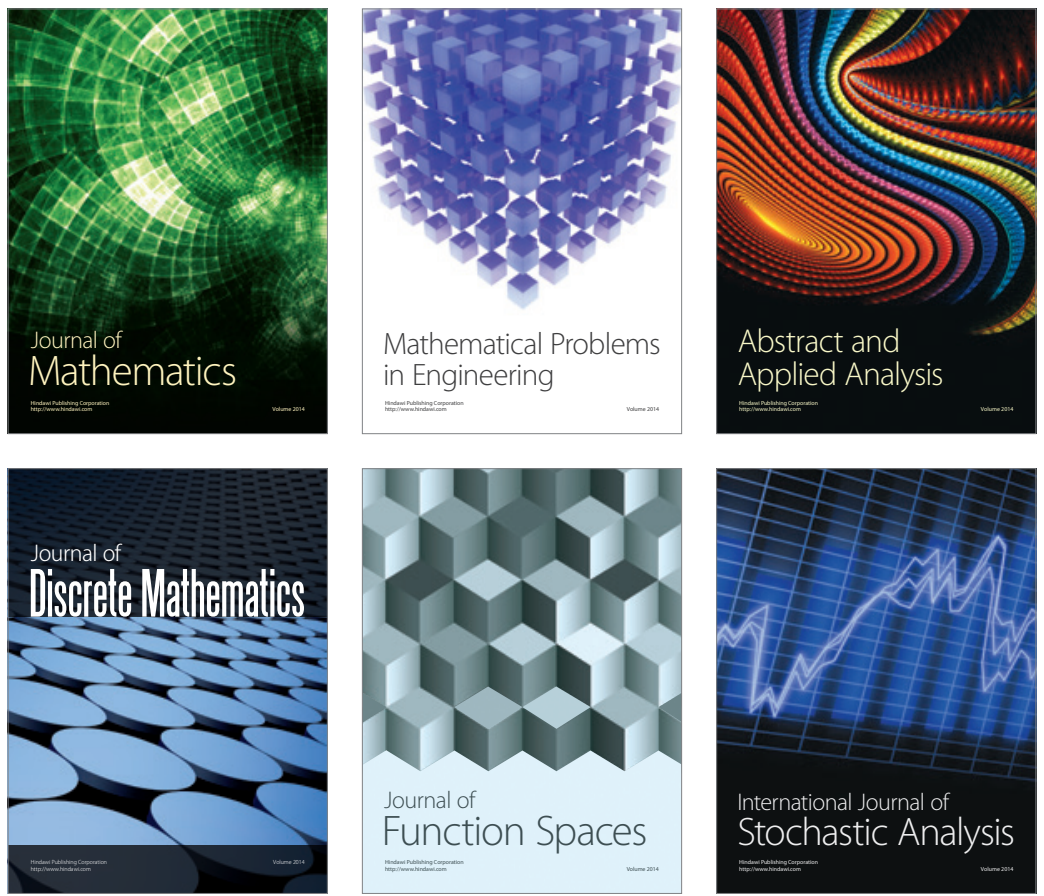

Journal of

Function Spaces

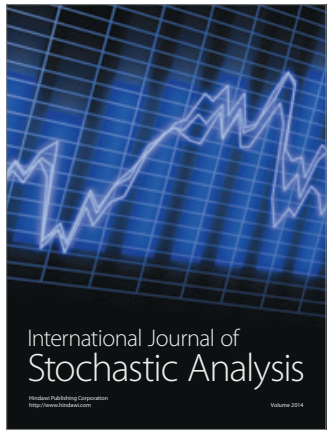

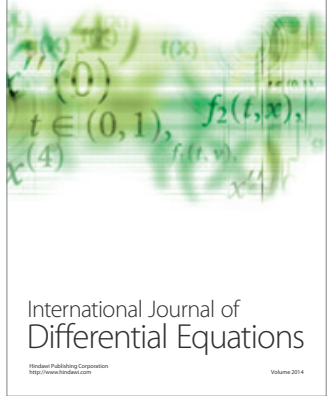
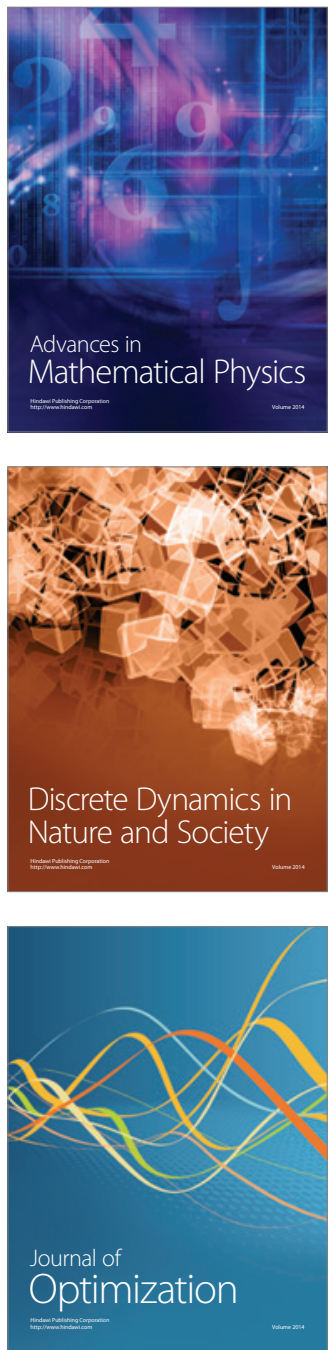